\title{
2 Qualitätsberichterstattung zur Hüft- und Knieendoprothetik in Österreich - wie Routinedaten zur Analyse von Revisionen genutzt werden
}

Silvia Türk, Martin Heidinger und Margarita Amon

\subsection{Einleitung}

Die Implantation von künstlichen Gelenken stellt heutzutage zunehmend einen Routineeingriff dar. Die epidemiologische Analyse von Primärimplantationen zeigt, dass Österreich bei der Häufigkeit von Hüfttotalendoprothesen (HTEP; 210 HTEP pro 100.000 Einwohner) und Knietotalendoprothesen (KTEP; 202 KTEP pro 100.000 Einwohner) im internationalen Spitzenfeld liegt (s. Abb. 1) (Sadoghi et al. 2012; Vielgut et al. 2013). Die meisten dieser Eingriffe finden in Österreich bei Patienten der Altersgruppe von 70-74 Jahren statt (Leitner et al. 2018).

Die Revisionshäufigkeit stieg im Zeitraum von 2009 bis 2015 sowohl bei HTEP als auch bei KTEP an (Leitner et al. 2018). Dies entspricht internationalen Entwicklungen (Bhandari et al. 2012; Boddapati et al. 2018; McGrory et al. 2016). Um dabei sowohl Patientenorientierung als auch Patientensicherheit zu garantieren, sind umfassende Qualitätsmanagementsysteme notwendig, die Qualität messbar und vergleichbar machen, Verbesserungspotenziale für Gesundheitsdienste- und Medizinprodukteanbieter aufzeigen und somit eine optimale Versorgung ermöglichen. Diese Systeme sollen im Sinne der österreichischen Qualitätsstrategie im Gesundheitswesen Ziele erfüllen, die unter anderem die kontinuierliche Verbesserung der Prozess- und Ergebnisqualität, die Transparenz im Gesundheitswesen und Veröffentlichung von Qualitätsberichten umfassen. 

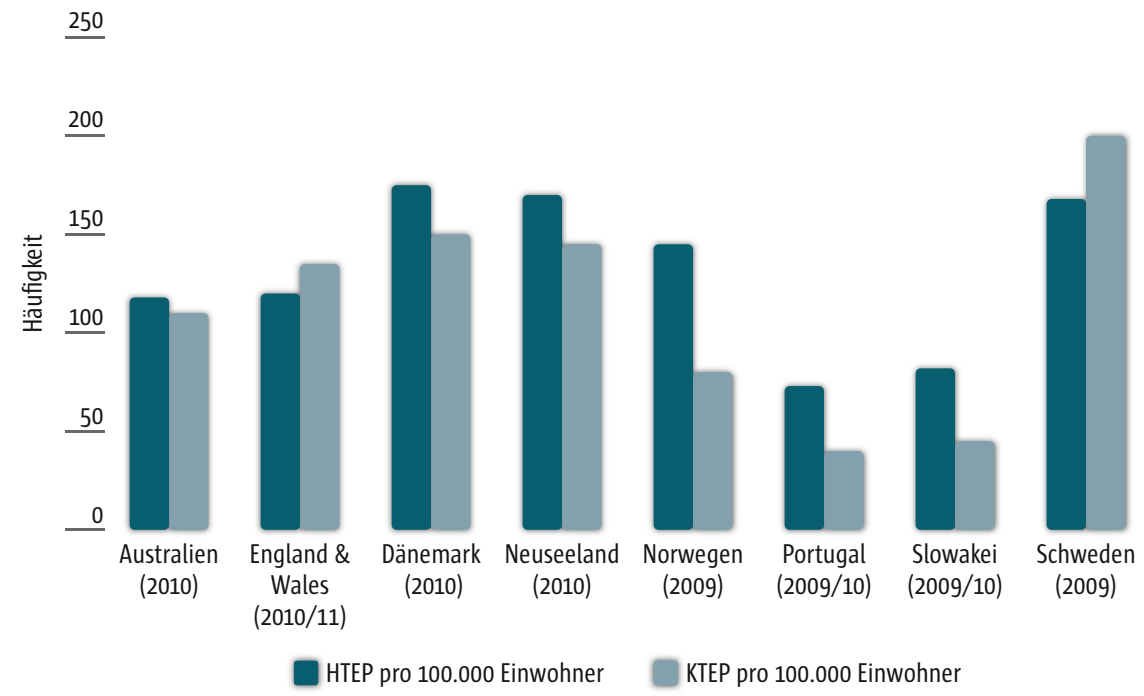

Abb. 1 Häufigkeit primärer Hüft- und Knietotalendoprothesen im internationalen Vergleich (adaptiert nach Sadoghi et al. 2012; Vielgut et al. 2013)

Die nationale Entwicklung ging seit 2001 von freiwilligen Registern für Hüftendoprothesen aus, die allerdings mit einer geringen Rücklaufquote verbunden waren. Weniger als 15\% der abgerechneten Fälle waren darin enthalten. Valide Daten als Grundlage für optimale Qualitätsarbeit waren daher nicht ausreichend vorhanden. In weiterer Folge wurde die Routine-Dokumentation im Zuge der leistungsorientierten Krankenanstaltenfinanzierung (LKF) um verpflichtende Routinedaten erweitert und in Einklang mit dem System Austrian Inpatient Quality Indicators (A-IQI) gebracht, das sich vollständig auf Routinedokumentation stützt. Dadurch werden die Daten für Diagnostik, Therapie, Implantation und Revision vollständig, einheitlich und österreichweit zu 100\% erfasst.

Der vorliegende Beitrag stellt unsere Erfahrungen und das Potenzial der vollständigen österreichweiten Erfassung von Hüft- und Knieendoprothesen-Revisionen auf Basis erweiterter Routinedaten dar. Zusätzlich stellen wir unseren Weg der Ergebnisdissemination und der praktischen Konsequenzen vor.

\subsection{Methodik der Datenerhebung}

Aufgrund der mangelnden Dateneingabe in den freiwilligen Registern entschied sich das nunmehrige österreichische Bundesministerium für Arbeit, Soziales, Gesundheit und Konsumentenschutz (BMASGK) 2013 in Zusammenarbeit mit den wissenschaftlichen Fachgesellschaften für Orthopädie und Unfallchirurgie dazu, die Datenerhebung umzugestalten. Das Ziel war es, die Registerinformationen daraufhin zum Großteil durch Routinedaten zu erfas- 
sen, um einen vollständigen und korrekten Datensatz zu schaffen. Dies geschah zusätzlich zu der Regelung der österreichischen Medizinproduktebetreiberverordnung (BGBl. II Nr. 70/2007 i.d.g.F.), wodurch jeder Betreiber eines Medizinproduktes ein dementsprechendes Implantatregister zu führen hat. Ein Großteil der erfassten Parameter wurde durch Erweiterungen und Differenzierungen in die Routinedokumentation des österreichischen LKF-Systems aufgenommen und als Pflichtfelder bei der Codierung definiert. Diese beinhaltet bspw. die Angabe zumindest eines Revisionsgrundes (aus 30) als Diagnose bei jeder Hüft- oder Knierevision oder eine Seitenangabe (li/re). Weiters wurden die Eingriffe an Hüfte und Knie in einer weitaus differenzierteren Form im LKF-Leistungskatalog abgebildet. Indikatoren für Erstimplantation und Revision wurden in A-IQI integriert. Die gesonderte Erfassung jedes Revisions-Falles erfolgt über eine Website. Alle bereits vorhandenen Routinedaten sind dort bereits vorbefüllt, und nur mehr die darüber hinaus zusätzlich benötigten Informationen sind vom Krankenhaus einzugeben. Diese ergänzenden, retrospektiv erhobenen Daten betreffen vor allem:

- Komorbiditäten

- Komplikationen

- Stehzeit und

- verwendete Medizinprodukte.

Wie auch in A-IQI erhalten sowohl die Gesundheitsfonds als auch die Krankenhäuser Ergebnisse und Vergleichswerte aus der Erhebung.

Durch die Neuregelung der Pseudonymisierung von Patientendaten im „Bundesgesetz über die Dokumentation im Gesundheitswesen “(BGBl. Nr. 745/1996 i.d.g.F.) im Jahr 2015 wurde die Messung von Revisionsraten ausschließlich durch Routinedaten erstmals möglich. Seither wird die Pseudonymisierung der gesundheitsbezogenen Daten der stationären Patientinnen und Patienten sowohl mittels Einweg-Ableitung des bereichsspezifischen Personenkennzeichens (bPK GH-CD) in ein Pseudonym als auch durch Einweg-Ableitung der Aufnahmezahl in eine Datensatz-ID sowie durch das Ersetzen des Geburtsdatums durch Altersgruppen gewährleistet.

Seit 2017 werden auch Revisionen von Knieendoprothesen detailliert in den Routinedaten des LKF-Systems dargestellt. Dementsprechend fand im selben Jahr die erste, über die Routinedaten hinausgehende bundesweite Erhebung aller Revisionen von Hüft- und Knieendoprothesen statt.

Die Basisauswertung erfolgt nach den von der Technischen Universität Berlin (Mansky et al. 2013, 2017) entwickelten „Inpatient Quality Indicators“ (IQI). Diese Qualitätsmessung wird analog zu Schweiz (CH-IQI) und Deutschland (G-IQI) nach Adaptation an nationale Gegebenheiten seit 2011 in ganz Österreich (A-IQI) verwendet (aktuell Vers. 5.1; Türk et al. 2018). Darüber hinaus werden Endoprothesen-Revisionen innerhalb von 12 Monaten nach Implantation differenziert nach Indikation und Eigen- sowie Fremdrevisionen analysiert. 
Zusätzlich zu den regulären A-IQI Indikatoren mit Datenbasis 2016 wurden alle in diesem Jahr durchgeführten Revisionen über die erweiterten und differenzierenden Routinedaten hinaus betrachtet - die gesonderte Erfassung jedes Revisions-Falles. Der Zeitpunkt der Erstimplantation ist in dieser Analyse unbeschränkt.

Die Erhebung von Revisionsraten ausschließlich mittels routinemäßig dokumentierten pseudonymisierten Patientendaten wurde durch das im Jahr 2015 in Kraft getretene „Bundesgesetz über die Dokumentation im Gesundheitswesen“ (BGBI. Nr. 745/1996 i.d.g.F.) erstmals möglich.

\subsection{Ergebnisse der Endoprothetikversorgung in Österreich}

\subsubsection{Erstimplantationen einer Hüft- oder Knieendoprothese}

Basierend auf den Routinedaten des österreichischen LKF-Systems wurden im Datenjahr 2016 in Österreich 21.988 Primärimplantationen an der Hüfte durchgeführt. Davon entfielen 16.854 Fälle $(76,65 \%)$ auf Erstimplantationen bei Koxarthrose und chronischer Arthritis, 4.536 Fälle $(20,63 \%)$ auf Erstimplantationen bei Schenkelhals- und pertrochantärer Fraktur und 598 Fälle (2,72\%) wurden unter „anderen“ Indikationen zusammengefasst.

Am Knie wurden im selben Datenjahr 19.443 Erstimplantationen durchgeführt. Davon entfielen 18.920 Fälle (97,31\%) auf Erstimplantationen bei Gonarthrose und chronischer Arthritis und 523 Fälle (2,69\%) wurden unter ,anderen“ Indikationen zusammengefasst.

Es wurden zusätzlich in 460 Fällen Tumorendoprothesen in Hüfte oder Knie implantiert. In 25 Fällen wurde einseitig eine Hüft- und Knieendoprothese implantiert.

\subsubsection{Revisionen einer Hüft- oder Knieendoprothese}

Wie im methodischen Teil dargestellt, basieren die hier dargestellten Daten sowohl auf erweiterten Routinedaten der LKF mit Datenbasis 2016 als auch auf retrospektiven Krankengeschichtenanalysen mit fallbezogenem Dokumentationsbogen. Festzuhalten ist, dass die Bezeichnung Revision in diesem Kapitel synonym zu Prothesenwechsel verwendet wird und keine Einschränkung des Erstimplantationsdatums gegeben ist.

Im Jahr 2016 wurden in Österreich demnach 2.521 Fälle einer Hüft- und 1.928 Fälle einer Knieendoprothese revidiert und erfasst.

Wesentliche epidemiologische Daten bei Hüft- und Knieendoprothesen-Revisionen sind in der Subgruppenanalyse der Tabelle 1 dargestellt. 
2 Qualitätsberichterstattung zur Hüft- und Knieendoprothetik in Österreich wie Routinedaten zur Analyse von Revisionen genutzt werden

Tab. 1 Detailergebnisse Hüft- und Knieendoprothesen-Revisionen, Datenbasis 2016 in Österreich*

\begin{tabular}{|c|c|c|}
\hline & $\begin{array}{l}\text { Hüftendoprothesen- } \\
\text { Revisionen }\end{array}$ & $\begin{array}{l}\text { Knieendoprothesen- } \\
\text { Revisionen }\end{array}$ \\
\hline Anzahl Revisionen (Datenbasis 2016) & 2.521 & 1.928 \\
\hline $\begin{array}{l}\text { Altersgruppen } \\
\begin{array}{l}\text { a } 50 \text { lahre } \\
\text { 51-60 Jahre } \\
\text { 61-70 Jahre } \\
\text { 71-80 Jahre } \\
\geq 80 \text { |ahre }\end{array}\end{array}$ & $\begin{array}{r}9,41 \% \\
15,31 \% \\
30,74 \% \\
34,07 \% \\
10,48 \% \\
\end{array}$ & $\begin{array}{r}9,79 \% \\
18,36 \% \\
35,01 \% \\
32,62 \% \\
4,20 \% \\
\end{array}$ \\
\hline mindestens 1 Komorbidität & $57,6 \%$ & $53,1 \%$ \\
\hline Polypharmazie (> 5 regelmäßige Medikamente/d) & $52 \%$ & $50,8 \%$ \\
\hline Übergewicht (BMI > 25) u. Adipositas & $65 \%$ & $81,2 \%$ \\
\hline Revisionen in der Klinik der Erstimplantation & $57,2 \%$ & $59,2 \%$ \\
\hline 3 häufigste Revisionsgründe & $\begin{array}{l}32,5 \% \text { Implantat- } \\
\text { lockerung } \\
21,9 \% \text { Infektion } \\
15,6 \% \text { Periprothet. } \\
\text { Fraktur }\end{array}$ & $\begin{array}{l}34,4 \% \text { Infektion } \\
24,2 \% \text { Implantat- } \\
\text { lockerung } \\
17,4 \% \text { sonstige }\end{array}$ \\
\hline $\begin{array}{l}\text { Prothesen-Lebensdauer** } \\
\text { 0-2 Jahre } \\
\text { 3-9 lahre } \\
\text { 10-19 lahre } \\
\text { über } 20 \text { lahre }\end{array}$ & $\begin{array}{l}38,0 \% \\
22,2 \% \\
29,1 \% \\
10,7 \%\end{array}$ & $\begin{array}{r}46,9 \% \\
31,8 \% \\
19,3 \% \\
2,0 \%\end{array}$ \\
\hline \multicolumn{3}{|c|}{$\begin{array}{l}\text { * Prozentzahlen teilweise gerundet } \\
\text { ** Die Prothesen-Lebensdauer ist definiert als Zeitraum zwischen Erstimplantation und Erst-Revision. Durch die } \\
\text { Rekonstruktion von Patientenhistorien war es möglich, die Prothesen-Lebensdauer einzelner Implantate zu er- } \\
\text { mitteln (n Hüfte }=1.523, n \text { Knie }=1.157 \text { ). Als Limitation muss hierbei allerdings ausdrücklich festgehalten werden, } \\
\text { dass lange Prothesen-Lebensdauern, bei denen die Rekonstruktion der Patientenhistorie nicht möglich war, } \\
\text { unterrepräsentiert sein können. Demnach besteht die Möglichkeit, dass die repräsentierten Daten hin zu kürzeren } \\
\text { Prothesen-Lebensdauern verschoben sind. }\end{array}$} \\
\hline
\end{tabular}

Mindestens eine Komorbidität war bei 57,56\% der Patientinnen und Patienten, die eine Hüftendoprothesen-Revision erhielten, sowie bei 53,1\% der Personen mit einer Knieendoprothesen-Revision vorhanden (s. Abb. 2).

Die präoperative Verweildauer von stationärer Aufnahme bis zum Eingriff betrug bei zwei Drittel der Hüftendoprothesen-Revisionen (66,89\%) und bei dem Großteil der Knieendoprothesen-Revisionen (80,86\%) zwischen o bis 2 Tagen. Die Verweildauer lag in 38,48\% der Hüftendoprothesen-Revisionen und in 47,20\% der Knieendoprothesen-Revisionen zwischen 8 bis 14 Tage.

urheberrechtlich geschützt, 


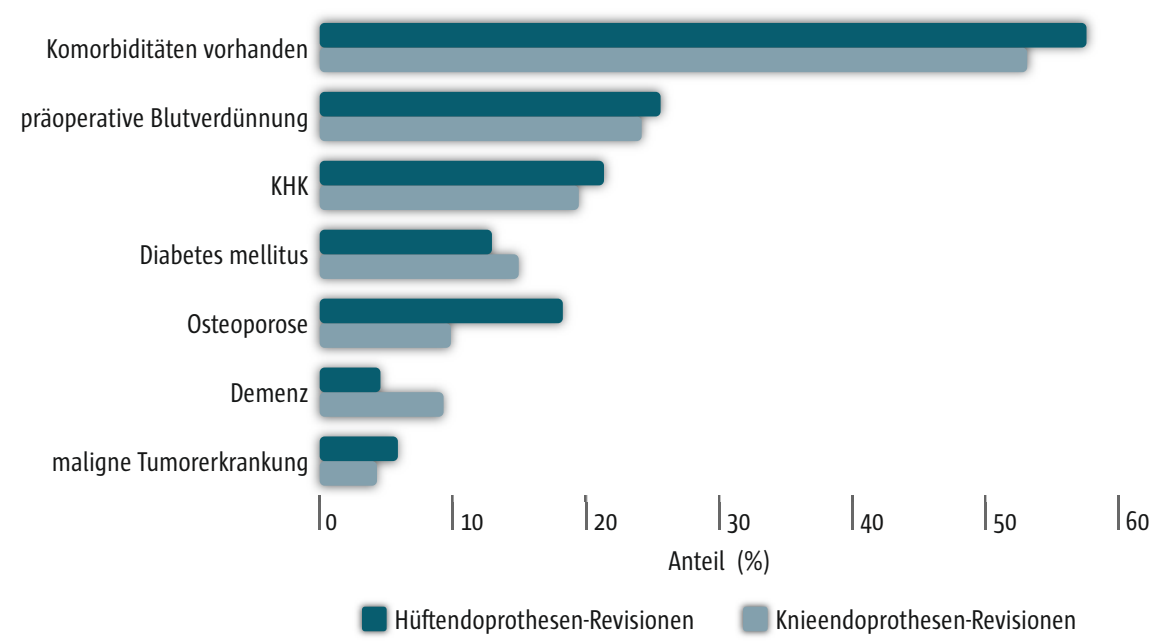

Abb. 2 Anteil von Patientinnen und Patienten mit Komorbiditäten bei Hüft- und Knieendoprothesen-Revisionen

\section{Wechseloperationen innerhalb eines Jahres - Ergebnisse der Sonderauswertung Endoprothetik-Revisionen}

Bei Hüftendoprothesen zeigte sich eine Revisionsrate innerhalb eines Jahres von 1,98\%. Der Großteil (93,12\%) der Revisionen einer Hüftendoprothese innerhalb eines Jahres wurde in derselben Krankenanstalt durchgeführt, in der auch die Erstimplantation erfolgte.

Bei Knieendoprothesen lag die Revisionsrate innerhalb eines Jahres bei 2,13\%. Auch bei einer Knieendoprothesen-Revision innerhalb eines Jahres wurde der Großteil (9o,66\%) in derselben Krankenanstalt durchgeführt, in der auch die Erstimplantation erfolgte.

\section{5/2016 wurden in Österreich rund 2\% der Hüft- als auch der Knie- endoprothesen-Primärimplantationen innerhalb eines Jahres revi- diert.}

\subsubsection{Gründe für Wechseloperationen}

Mittels der oben präsentierten Daten der bundesweiten Erhebung wurde in einer Korrelationsanalyse der Zusammenhang zwischen unterschiedlichen Messgrößen und Patientinnen-/Patienten-Eigenschaften untersucht und hinsichtlich der statistischen Signifikanz mit einem Konfidenzintervall von 95\% $(\mathrm{p} \leq 0,05)$ als auch der Effektstärke untersucht.

urheberrechtlich geschützt, 
2 Qualitätsberichterstattung zur Hüft- und Knieendoprothetik in Österreich wie Routinedaten zur Analyse von Revisionen genutzt werden

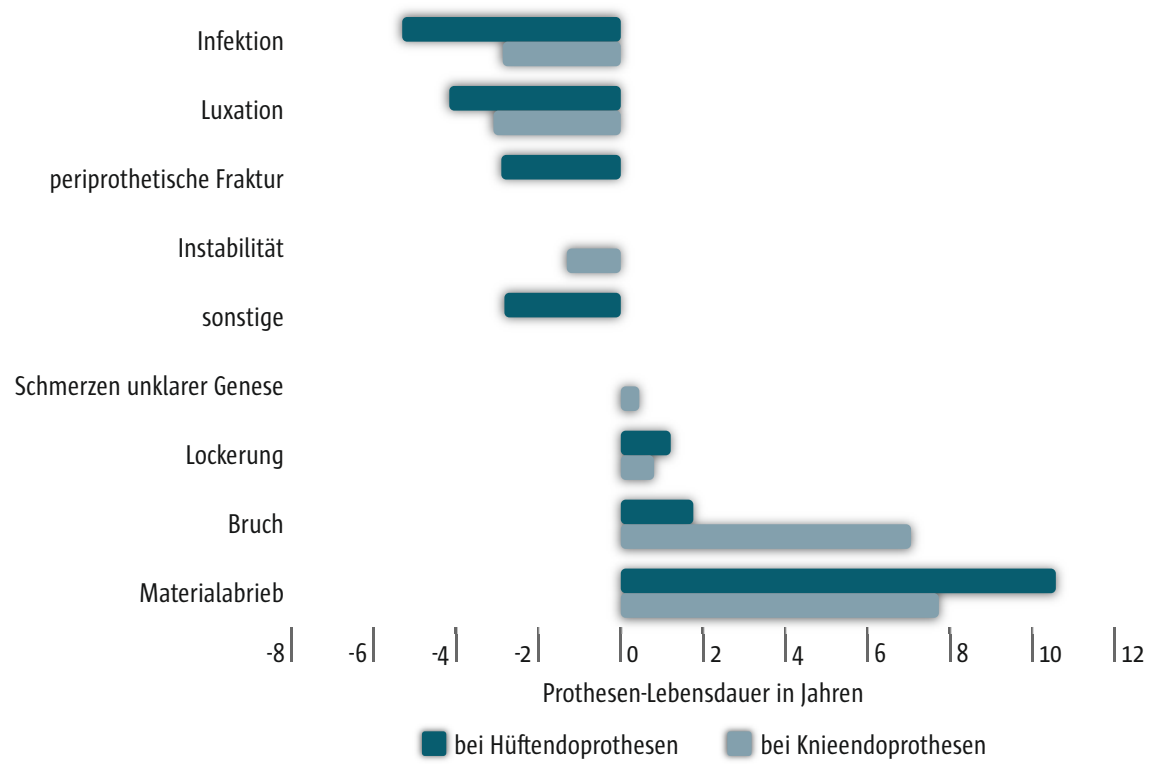

Abb. 3 Signifikante Zusammenhänge zwischen Prothesen-Lebensdauer und Hauptrevisionsgründen bei Hüft- und Knieendoprothesen-Revisionen. Die präsentierten Zusammenhänge sind allesamt statistisch signifikant $(p \leq 0,05)$ und zeigen sowohl den Zusammenhang als auch die Stärke des Zusammenhangs in Jahren an, der zwischen den jeweiligen Hauptrevisionsgründen und der damit verbundenen Prothesen-Lebensdauer im Vergleich zur durchschnittlichen Prothesen-Lebensdauer aller Hüft- respektive Knieendoprothesen besteht.

\section{Zusammenhang zwischen Hauptrevisionsgründen und Prothesen-Lebensdauer}

Die statistische Signifikanz wurde mittels eines zweiseitigen Wilcoxon-Rangsummentests ermittelt. Die Effektstärke wurde definiert als Differenz der Prothesen-Lebensdauer im Zusammenhang mit den Hauptrevisionsgründen. Es fanden sich signifikante Verkürzungen und Verlängerungen der Prothesen-Lebensdauern bei diversen Hauptrevisionsgründen, wie in Abbildung 3 dargestellt.

Exemplarisch soll die Entwicklung der Prothesen-Lebensdauern bei dem Hauptrevisionsgrund Infektion vorgestellt werden. So fand sich dabei eine signifikant kürzere Prothesen-Lebensdauer von Hüftendoprothesen, die im Mittel 5,28 Jahre betrug. Bei Knieendoprothesen betrug die signifikant verkürzte Prothesen-Lebensdauer durchschnittlich 2,88 Jahre.

\section{Komplikationen bei Wechseloperationen}

Die statistische Signifikanz wurde mittels Chi-Quadrat Test ermittelt. Die Effektstärke wurde durch das relative Risiko einer Komplikation angegeben. Komplikationen wurden im Zuge der Erarbeitung des A-IQI-Rückmeldebogens im Expertenkonsensus definiert. Folgende Komplikationen wurden erfasst: 
- allgemeine Komplikationen (TVT, Herzinfarkt/-insuffizienz, Blutverlust, Allergien/Unverträglichkeiten, systemischer Infekt)

- OP-spezifische Komplikationen (lokaler Infekt, intraoperative Fraktur, lokale Blutung/Hämatom, Nervenverletzung, Luxation, Lockerung)

Eine signifikante Erhöhung des Komplikationsrisikos fand sich bei dem Hauptrevisionsgrund Infektion. Dabei erhöhte sich das relative Risiko für eine Komplikation bei Hüftendoprothesen-Revisionen auf das 2,33-fache und bei Knieendoprothesen-Revisionen auf das 2,26-Fache.

\subsection{Nutzung der Qualitätsdaten}

Durch Qualitätsmessung kann Verbesserungspotenzial in der gesamten Patientenbehandlung (Strukturen, Prozesse, Ergebnisse) identifiziert werden. Für den Umgang mit den vorhandenen Qualitätsdaten wurde ein Jahresablaufplan erarbeitet, der die Meilensteine Datenanalyse (Erstanalyse, Versand der Ergebnisse an Gesundheitsfonds und Krankenhäuser, Kodierüberprüfung), Information und Peer-Review-Verfahren beinhaltet.

Um die Ergebnisse der Messungen zu disseminieren werden die Resultate im Wesentlichen für zwei Gruppen aufbereitet: einerseits für Expertinnen und Experten (A-IQI Bericht), andererseits für die Bevölkerung (kliniksuche.at).

\subsubsection{Nationaler A-IQI-Qualitätsbericht und Themenbericht Endoprothetik}

Seit 2013 erfolgt durch das BMASGK eine jährliche Berichterstattung hinsichtlich des Systems A-IQI. Dargestellt werden im A-IQI-Bericht die Ergebnisse der A-IQI-Qualitätsindikatoren des jeweiligen Berichtsjahres, die Ergebnisse der stattgefundenen Peer-Review-Verfahren sowie die sich daraus ableitenden Verbesserungsmaßnahmen und deren Monitoring. Schlussendlich wird ein Ausblick über die zukünftigen Aktivitäten gegeben (Türk et al. 2018a).

In Spezialberichten, wie beispielsweise dem kürzlich veröffentlichten Bericht zur „Hüft- und Knieendoprothetik in Österreich“, wird auf die historische Entwicklung und die gesetzlichen Crundlagen im speziellen Themengebiet der Endoprothetik eingegangen. Neben Beiträgen durch die Fachgesellschaften für Orthopädie und Unfallchirurgie werden darin auch die Perspektiven der Industrie und der Patientenanwaltschaft dargestellt. Schlussendlich werden die Ergebnisse der Hüft- und Knieendoprothetik im Detail dargestellt (Türk et al. 2018b).

Die Berichterstattung für Expertinnen und Experten erfolgt einerseits mit den jährlichen A-IQI-Berichten sowie andererseits mit Spezialberichten, wie beispielsweise dem kürzlich veröffentlichten Bericht zur „Hüft- und Knieendoprothetik in Österreich“. 


\subsubsection{Peer-Review-Verfahren}

Auslöser für Peer-Review-Verfahren sind die vorhandenen Qualitätsdaten. Jährlich werden Schwerpunkte festgelegt, aktuell Magenoperationen. Alle statistisch signifikanten Auffälligkeiten in den Indikatoren werden laut Jahresablaufplan analysiert und Peer-Review-Verfahren durchgeführt. Das Herzstück des Verfahrens ist die gemeinsame Diskussion der Einzelfälle mit den Verantwortlichen vor Ort und eine anschließende gemeinsame Festlegung von Verbesserungsmaßnahmen. Ziele sind:

- Optimierung des gesamten Behandlungsprozesses (von Abteilungsdenken zu interdisziplinären Fallbetrachtungen; Abläufe, Strukturen und Schnittstellen optimieren)

- Aufdecken lokaler Besonderheiten (Krankenhausstruktur und Umfeld)

- Etablieren einer offenen Fehlerkultur (offene sachliche Diskussion mit allen Beteiligten, Förderung von kritischer Selbsteinschätzung)

- Nachhaltigkeit des Verbesserungsprozesses

- Kontrolle der Kennzahlen

\subsubsection{Kliniksuche.at - Veröffentlichung der Ergebnisse für die Bevölkerung}

Kliniksuche. at ist eine vom BMASGK unterhaltene und mit Daten der LKF sowie Daten der Plattform Qualitätsberichterstattung befüllte Online-Plattform zur Veröffentlichung von patientenrelevanten Qualitätsdaten. Durch die Darstellung soll die Bevölkerung in Vorbereitung auf einen Krankenhausaufenthalt bei der Entscheidungsfindung unterstützt werden.

Die dargestellten Informationen auf Kliniksuche. at umfassen sieben Themengebiete mit insgesamt 35 einzeln abrufbaren Eingriffen, die österreichweit auf Krankenhausebene dargestellt werden. Konkret werden dazu die Anzahl der behandelten Fälle, diverse Kriterien zum Aufenthalt (z.B.: Verweildauer, Operationstechnik) als auch allgemeine Kriterien des Krankenhauses dargestellt.

\section{Die staatlich verwaltete Online-Plattform kliniksuche.at veröffentlicht patientenrelevante Qualitätsdaten und unterstützt die Bevölkerung dadurch sowohl bei der Vorbereitung eines Krankenhausaufenthaltes als auch bei der Entscheidungsfindung.}

Im Themengebiet „Bewegungsapparat“, werden unter anderem die elektiven Eingriffe „Hüftprothese“ und „Knieprothese“ aufgeführt. Angezeigt werden darin sämtliche Krankenhäuser in denen diese Operationen durchgeführt werden. Dargestellt und bewertet wird die Anzahl dieser Operationen (über, in oder unter der bundesweiten Bandbreite) und als Kriterium für den Aufenthalt die Verweildauer (bundesweiter Vergleichswert) im jeweiligen Kranken- 


\section{(2) KLINIKSUCHE.at}

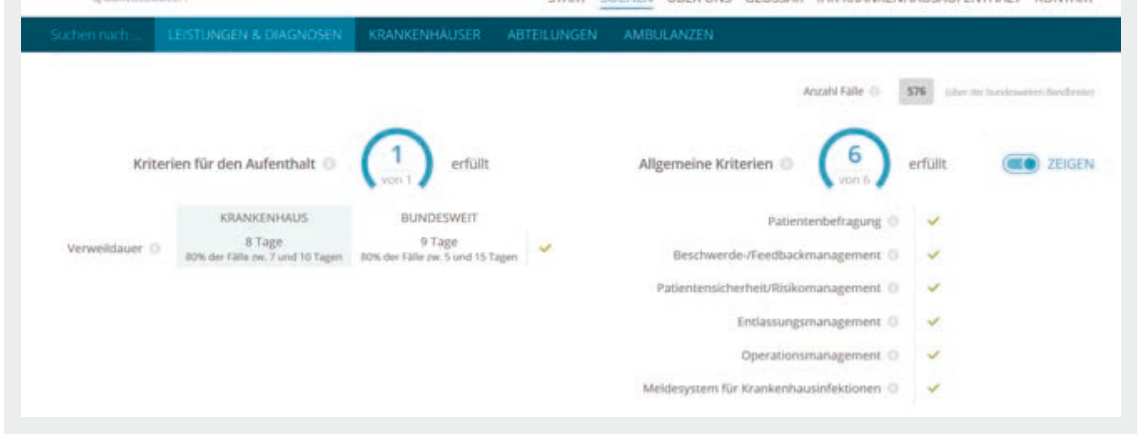

Abb. 4 Darstellung eines Beispielkrankenhauses auf kliniksuche.at (Quelle: kliniksuche.at)

haus. Allgemeine Kriterien des Krankenhauses umfassen Faktoren zu eingerichtetem Entlassungs- und OP-Management, Meldesystem für Krankenhausinfektionen, Patientenbefragungen, Beschwerde-/Feedbackmanagement und strukturiertem Vorgehen im Umgang mit Risiken, kritischen Situationen und Fehlern (s. Abb. 4).

\subsection{Zusammenfassung}

Ansteigende Erst- und Folgeimplantationen (Revisionen, Wechsel) von Knieund Hüftendoprothesen bedürfen eines besonderen Monitorings inkl. der Evaluation aller Qualitätsmerkmale.

In Österreich erfolgt die Diagnosencodierung entsprechend internationaler Vorgaben nach ICD-10. Die leistungsorientierte Krankenanstaltenfinanzierung (LKF) erlaubt durch eine jährliche Anpassung über eine nationale Verordnung eine rasche Anpassung an neue Gegebenheiten. Diese bundeseinheitliche, verpflichtende und einfache Codierung im Rahmen der Abrechnung einerseits, und die Pseudonymisierung von Patientendaten andererseits, ergeben zusammen eine kurzfristig verfügbare (ca. 6 Monate nach Kalenderjahresabschluss) und praktisch komplette österreichweite Datenlage und ist Registern damit in der Qualitätsarbeit überlegen. Kurzfristige Revisionen (in unserer Auswertung innerhalb von 12 Monaten) von Implantaten können dadurch identifiziert sowie Langzeitergebnisse, Korrelationsanalysen und eventuelle Fehleranalysen durchgeführt werden. Eine Auswertung anhand von A-IQI-Indikatoren und ergänzende Vor-Ort-Peer-Review-Verfahren erlauben eine unmittelbare individuelle Analyse einer optimierungsbedürftigen Prozess- und Ergebnisqualität, sofortige Reaktion und weiters ein Monitoring eingeleiteter Maßnahmen. Analog dazu können, neben der „akuten“ Fehlermeldung von Medizinprodukten an das nationale Bundesamt für Sicherheit im Gesundheitswesen, auch langfristig eventuelle Schwachstellen bei eingesetzten Medizinprodukten erkannt und korrigiert werden.

urheberrechtlich geschützt, 
Die erhobenen Daten können zu bundesweiten und internationalen Vergleichen verwendet werden. Durchschnittsberechnungen für ganz Österreich erlauben Leistungserbringern und auch Patientinnen und Patienten individuelle Vergleiche. Eine 2013 publizierte systematische Analyse nationaler Endoprothetik-Register zeigte in Bezug auf Revisionsursachen bei HTEP vorwiegend aseptische Lockerungen, gefolgt von Luxationen und septischen Lockerungen. Bei KTEP fanden sich ebenso primär aseptische Lockerungen, gefolgt von septischen Lockerungen und Schmerzen aufgrund unklarer Ursachen als Revisionsgründe (Sadoghi et al. 2013). Auch in Österreich ist bei Hüftendoprothesen-Revisionen 2016 der primäre Revisionsgrund die nicht infektiöse Lockerung, bei den Knieendoprothesen-Revisionen sind in Österreich die Infektionen (septische Lockerung) häufiger als international. Dies ist in Hinblick auf die erhöhte postoperative Morbidität und Mortalität nach Implantatinfektionen von Bedeutung (Boddapati et al. 2018).

Die Kombination aus erweiterten, differenzierenden und verpflichtend mit der Abrechnung zu erfassenden Codes, die Auswertung anhand von A-IQI-Indikatoren und Peer-Review-Verfahren erhöhen die Patientensicherheit und schaffen Transparenz für Patientinnen und Patienten, Gesundheitsdiensteund Medizinprodukteanbieter. Durch die Verbindung der Datenerfassung mit der Leistungsabrechnung sind die Daten bundesweit gleichartig und vollständig erfasst. Sie lassen dadurch hohe Integrität mit minimalem administrativem Aufwand erwarten.

Ab 1.1.2015 waren die gesetzlichen Grundlagen der Verwendung pseudonymisierter Daten geschaffen, die eine exakte Darstellung aller Krankheitsverläufe (Patientenkarrieren) ermöglicht. Dies wurde für die oben dargestellte Sonderauswertung zu Endoprothesenrevisionen innerhalb der ersten 12 Monate nach Erstimplantation verwendet und zeigt, dass eine klare statistische Datenlage entsteht. Seit ihrer Einführung 2015 erlaubt die Pseudonymisierung der Patientendaten die Betrachtung von Jahr zu Jahr längerer Krankheitsverläufe und reduziert zusätzliche retrospektive Erhebungen.

\section{Take home messages}

- Durch die Integration relevanter Parameter der Hüft- und Knie-Endoprothetik in das System A-IQI sowie in die österreichische leistungsorientierte Krankenanstaltenfinanzierung (LKF), durch die damit einhergehende Reduktion zusätzlicher retrospektiver Erhebungen je Revision sowie durch die Neuregelung der Pseudonymisierung von Patientendaten wurde die 100\%ige Erfassung aller nationalen Revisionen von Hüft- und Knietotalendoprothesen ermöglicht.

- Als häufigste Hauptrevisionsgründe zeigten sich sowohl bei Hüft-als auch bei Knieendoprothesen Implantatlockerungen und Infektionen. Mittels Korrelationsanalysen konnte weiters gezeigt werden, dass signifikante Zusammenhänge zwischen diversen Hauptrevisionsgründen 
und der Haltbarkeit von Hüft- und Knieimplantaten bestehen. Infektionen verkürzten die durchschnittliche Haltbarkeit einer Hüftendoprothese demnach um 5,28 Jahre, die Haltbarkeit einer Knieendoprothese um 2,88 lahre.

- Neben der Berichterstattung für Expertinnen und Experten wird die Aufbereitung der Qualitätsdaten in einer patientenrelevanten und verständlichen Form auf der staatlich verwalteten Online-Plattform kliniksuche.at regelmäßig durchgeführt. Dadurch soll die Bevölkerung bei der Vorbereitung auf einen Krankenhausaufenthalt und der Entscheidungsfindung Unterstützung erhalten.

\section{Literatur}

Bhandari M, Smith J, Miller L, Block I (2012) Clinical and Economic Burden of Revision Knee Arthtroplasty. Clinical Medicine Insights: Arthritis and Musculosceletal Disorders 5:89-94. doi: 10.4137/CMAMD.S10859

Boddapati V, Fu MC, Mayman DI, Su EP, Sculco PK, McLawhorn AS (2018) Revision total knee arthroplasty for periprosthetic joint infection is associated with increased postoperative morbidity and mortality relative to noninfectious revisions. I Arthroplasty. 33(2):521-526. doi: 10.1016/j.arth.2017.09.021.

Leitner L, Türk S, Heidinger M, Stöckl B, Posch F, Maurer-Ertl W, Leithner A, Sadoghi P (2018) Trends and Economic Impact of Hip and Knee Arthroplasty in Central Europe: Findings from the Austrian National Database. Sci Rep. 8(1):4707. doi: 10.1038/s41598-018-23266-w

Mansky T, Nimptsch U, Cools A, Hellerhoff F (2017) G-IQI-German Inpatient Quality Indicators Version 5.1Band 2: Definitionshandbuch für das Datenjahr 2017. Universitätsverlag der TU Berlin (Version 4.0: 2013)

Mansky T, Nimptsch U, Winklmair C, Hellerhoff F (2013) G-IQI German Inpatient Quality Indicators Version 4.0. Band 3. Erläuterungen und Definitionshandbuch für das Datenjahr 2013. 2. Auflage. Universitätsverlag der TU Berlin. URL: https://www.seqmgw.tu-berlin.de/fileadmin/fg241/GIQI_V40_Band_3_Aufl_2_online.pdf (abgerufen am 26.09.2018)

McGrory B, Etkin C, Lewallen D (2016) Comparing contemporary revision burden among hip and knee joint replacement registries. Arthroplasty Today 2, 83e86. http://dx.doi.org/10.1016/j.artd.2016.04.003

Sadoghi P, Liebensteiner M, Agreiter M, Leithner A, Böhler N, Labek G (2013) Revision surgery after total joint arthroplasty: a complication-based analysis using worldwide arthroplasty registers. J Arthroplasty. 28(8):1329-32. doi: 10.1016/j.arth.2013.01.012.

Sadoghi P, Schröder C, Fottner A, Steinbrück A, Betz 0, Müller PE, Jansson V, Hölzer A (2012) Application and survival curve of total hip arthroplasties: a systematic comparative analysis using worldwide hip arthroplasty registers. Int Orthop. 36(11):2197-203. doi: 10.1007/s00264-012-1614-6.

Türk S, Amon M, Bachinger G, Heidinger M, Klimek P, Lindinger P, Vukic I, Wiss. Ges. f. Orthopädie und Unfallchirurgie (2018b) Hüft- und Knie-Endoprothetik in Österreich. Wien: BMASGK. https://www.bmgf.gv.at/ cms/home/attachments/7/5/0/CH1367/CMS1385999778812/180727_endoprothetik-bericht_final.pdf (abgerufen am 28.07.2018)

Türk S, Amon M, Pesec B, Rath I, Vukic I (2018) Austrian Inpatient Quality Indicators (A-IQI). Indikatorenbeschreibung Version 5.1. Wien: BMASGK. https://www.bmgf.gv.at/cms/home/attachments/3/2/9/CH1367/ CMS1411031157954/indikatorenbeschreibung_aiqi_5.1.pdf (abgerufen am 28.07.2018)

Türk S, Pesec B, Amon M, Rath I, Vukic I (2018a) Austrian Inpatient Quality Indicators (A-IQI) Bericht 2017. Wien: BMASGK. https://www.bmgf.gv.at/cms/home/attachments/7/5/0/CH1367/CMS1385999778812/ a-iqi_bericht_2017.pdf (abgerufen am 28.07.2018)

Vielgut I, Kastner N, Pichler K, Holzer L, Glehr M, Gruber G, Leithner A, Labek G, Sadoghi P (2013) Application and surgical technique of total knee arthroplasties: a systematic comparative analysis using worldwide registers. Int Orthop. 37(8):1465-9. doi: 10.1007/s00264-013-1933-2.

urheberrechtlich geschützt, 


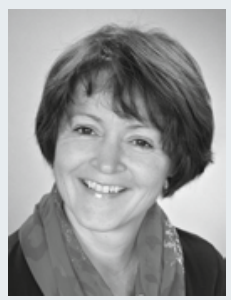

\section{Dr. Silvia Türk}

Lehre der pharmazeutischen Assistentin, Studium der Humanmedizin an der Universität Wien, Berechtigung zur selbstständigen Berufsausübung als Allgemeinmedizinerin, Studium Gesundheitsmanagement und -ökonomie an der Wirtschaftsuniversität Wien. Seit 2009 Abteilungsleiterin der Abteilung VIII/C/8, Qualität im Gesundheitswesen und Gesundheitssystemforschung des Bundesministeriums für Arbeit, Soziales, Gesundheit und Konsumentenschutz (BMASGK).

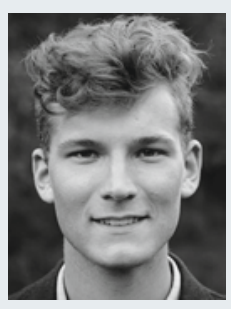

\section{Martin Heidinger}

Student der Humanmedizin an der Medizinischen Universität Graz und seit 2017 Mitarbeiter der Abteilung VIII/C/8 des Bundesministeriums für Arbeit, Soziales, Gesundheit und Konsumentenschutz (BMASGK).

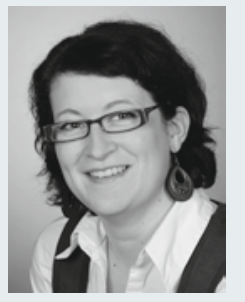

Margarita Amon, M.Sc.

Ausgebildete Gesundheits- und Krankenschwester. Studium Gesundheitsmanagement sowie Patientensicherheit und Qualität. Seit 2008 im Qualitätsmanagement tätig und seit 2014 Mitarbeiterin der Abteilung VIII/C/8 des Bundesministeriums für Arbeit, Soziales, Gesundheit und Konsumentenschutz (BMASGK). 